\title{
GLUEBALL MASS PREDICTIONS OF THE VALENCE APPROXIMATION TO LATTICE QCD
}

\author{
H. Chen, J. Sexton円, A. Vaccarino, \\ and D. Weingarten \\ IBM Research \\ P.O. Box 218, Yorktown Heights, NY 10598
}

\begin{abstract}
We evaluate the infinite volume, continuum limit of glueball masses in the valence (quenched) approximation to lattice QCD. For the lightest states with $J^{P C}$ of $0^{++}$and $2^{++}$, we obtain $m_{0}=1340 \pm 160 \mathrm{MeV}$ and $m_{2}=1900 \pm 320 \mathrm{MeV}$.
\end{abstract}

\footnotetext{
${ }^{1}$ permanent address: Department of Mathematics, Trinity College, Dublin 2, Republic of Ireland
} 


\section{INTRODUCTION}

Although dozens of observed hadrons have been convincingly interpreted as bound states of combinations of quarks and antiquarks, physical particles composed primarily of chromoelectric field, glueballs, have so far not been identified unambiguously in experiment. One possible explanation is that glueballs simply may not exist. All excitations of the chromoelectric field might occur only mixed with a large number of quark-antiquark states. Each mixed state might have only a small probability amplitude of excited chromoelectric field and, as a result, show no easily identifiable experimental signature of the presence of a field excitation. Alternatively, some of the particles already observed in experiment may actually be glueballs and may not have been identified because QCD's predictions for glueball masses and decays have not yet been determined accurately enough.

In the present article, we evaluate the infinite volume, continuum limit of the predictions of lattice QCD, in the valence approximation, for the masses of the lightest glueballs with $J^{P C}$ of $0^{++}$and $2^{++}$. The $0^{++}$is probably the lightest member of the glueball spectrum and the $2^{++}$the next lightest [1, 2]. The valence approximation may be viewed as replacing the momentum dependent color dielectric constant arising from quark-antiquark vacuum polarization with its zero-momentum limit [3]. For glueball masses, this approximation amounts to a reinterpretation of the predictions of pure gauge theory. Pure gauge QCD certainly predicts the existence of unmixed glueballs.

The valence approximation could be expected to be fairly reliable for low-lying hadron masses, which are determined largely by the low momentum behavior of the chromoelectric field. This expectation is supported by a recent valence approximation calculation [1] of the masses of eight low-lying baryons and quark-antiquark mesons. The predicted masses are all within $6 \%$ of experiment and all differences between prediction and experiment are consistent with the calculation's statistical uncertainties. The prediction we obtain here for the $0^{++}$glueball mass, $1340 \pm 160 \mathrm{MeV}$, lies below the largest mass, $m_{\Omega}=1672 \mathrm{MeV}$, for which the valence approximation has now, to some degree, been established as reliable. Allowing a disagreement of up to $80 \mathrm{MeV}$,

$6 \%$ of $1340 \mathrm{MeV}$, between the valence approximation and full QCD, the predicted 
$0^{++}$mass is consistent with the masses of either of two well-established $0^{++}$states,

$f^{0}(1400)$ and $f^{0}(1590)$. The mass prediction for the $2^{++}$glueball, $1900 \pm 320 \mathrm{MeV}$, is close enough to the range for which the valence approximation has been tested that it appears reasonable to expect a disagreement of at most $6 \%$ with full QCD in this case also, giving an additional uncertainty of $120 \mathrm{MeV}$. The overall uncertainty in the $2^{++}$mass is large enough that this state could be any one of a half dozen or so observed resonances.

Infinite volume, continuum limit glueball masses obtained from the valence approximation to lattice QCD have now also been reported in Ref. [5], which appeared while the present work was in progress. The predicted $0^{++}$and $2^{++}$masses are $1550 \pm 50 \mathrm{MeV}$ and $2270 \pm 100 \mathrm{MeV}$, respectively. The prediction for the $0^{++}$mass is about 1.3 standard deviations above our result. It seems to us that the central values of both predictions may be somewhat larger and the error bars of both predictions somewhat smaller than the data reported in Ref. [5] actually suggests. We will return to this subject below.

The calculations described here were done on the GF11 parallel computer at IBM Research [6] and took about six months to complete. GF11 was used in configurations ranging from 375 to 480 processors, with sustained speeds ranging from 5 Gflops to 7 Gflops.

\section{DEFINITIONS AND ALGORITHMS}

To define propagators from which light glueball masses can be extracted efficiently, it is useful to form smeared gauge fields with reduced coupling to the underlying gauge field's high momentum components [7, 8]. In place of the gauge invariant smearing of Refs. [7, 8], however, we use a smearing method based on Coulomb gauge. Each gauge configuration on which glueball propagators are to be constructed is transformed to lattice Coulomb gauge, defined to maximize at each site the target function $\sum_{x i} \operatorname{Re} \operatorname{Tr}\left[U_{i}(x)\right]$, where the sum is over all lattice sites and space direction gauge links. A gauge transformation which produces a local maximum of this sum is found by a method qualitatively similar to the Cabbibo-Marinari-Okawa Monte Carlo 
algorithm. The lattice is swept repeatedly, and at each site we maximize the target function first by a gauge transformation in the $S U(2)$ subgroup of $S U(3)$ acting only on gauge index values 1 and 2, then by a gauge transformation in the $S U(2)$ subgroup acting only on index values 2 and 3, then by a gauge transformation in the subgroup acting only on index values 1 and 3. Maximizing the target function over $S U(2)$ subgroups is easier to program than a direct maximization over all of $S U(3)$. On the other hand, it is not clear that maximizing each site over $S U(3)$ would significantly accelerate the full transformation to Coulomb gauge. A local maximum is reached when at each site the quantity $R(x)$ vanishes where

$$
\begin{aligned}
& Q(x)=\sum_{i}\left[U_{i}(x)-U_{i}^{\dagger}(x-\hat{i})\right], \\
& R(x)=Q(x)-Q^{\dagger}(x)-\frac{2}{3} \operatorname{Im} \operatorname{Tr}[Q(x)] .
\end{aligned}
$$

The vector $\hat{i}$ is a unit lattice vector in the positive $i$ direction. We stop the iteration process when the sum over the lattice of the quantity $\operatorname{Tr}\left[R^{\dagger}(x) R(x)\right]$ becames smaller than a convergence parameter $r$.

Coulomb gauge smeared link variables $U_{i}^{s}(x)$ for $s \geq 1$ are then defined by the average

$$
U_{i}^{s}(x)=\frac{1}{(s+1)^{2}} \sum_{0 \leq p, q \leq s} U_{i}(x+p \hat{j}+q \hat{k}),
$$

where $j$ and $k$ are the two space directions orthogonal to $i$. The product of $s$ sequential $U_{i}^{s}(y)$ gives $V_{i}^{s}(x)$,

$$
V_{i}^{s}(x)=U_{i}^{s}(x) \ldots U_{i}^{s}[x+(s-1) \hat{i}] .
$$

From the $V_{i}^{s}(y)$ we define smeared loops $L_{i j}^{s}(x)$

$$
L_{i j}^{s}(x)=\operatorname{Tr}\left[V_{i}^{s}(x+s \hat{i}) V_{j}^{s}(x+2 s \hat{i}+s \hat{j}) V_{i}^{s}(x+s \hat{i}+2 s \hat{j})^{\dagger} V_{j}^{s}(x+s \hat{j})^{\dagger}\right] .
$$

This definition fits together as smoothly as possible the products of smeared links forming each side of $L_{i j}^{s}(x)$. The sum of $L_{i j}^{s}(x)$ over all $x$ in a hyperplane with fixed time component $t$ defines the zero-momentum loop variable $\bar{L}_{i j}^{s}(t)$. 
For smearing size 0, the loop variable $L_{i j}^{0}(x)$ is defined to be an unsmeared plaquette

$$
L_{i j}^{0}(x)=\operatorname{Tr}\left[U_{i}(x) U_{j}(x+\hat{i}) U_{i}(x+\hat{j})^{\dagger} U_{j}(x)^{\dagger}\right]
$$

and $\bar{L}_{i j}^{0}(x)$ is then defined from $L_{i j}^{0}(x)$.

A field for the $0^{++}$glueball, transforming according to the $A_{1}$ representation of the cubic symmetry group [四], is given by

$$
A^{s}(t)=\sum_{i j} \operatorname{Re}\left[\bar{L}_{i j}^{s}(t)\right]
$$

Fields for the $2^{++}$glueball, transforming according to the $E$ representation of the cubic symmetry group, are given by

$$
\begin{aligned}
& E_{1}^{s}(t)=\operatorname{Re}\left[\bar{L}_{12}^{s}(t)-\bar{L}_{23}^{s}(t)\right] \\
& E_{2}^{s}(t)=\operatorname{Re}\left[\bar{L}_{12}^{s}(t)+\bar{L}_{23}^{s}(t)-2 \bar{L}_{13}^{s}(t)\right] / \sqrt{3}
\end{aligned}
$$

From these glueball fields, we define glueball propagators as

$$
\begin{aligned}
& C_{0}^{s s^{\prime}}(t)=<A^{s}(t) A^{s^{\prime}}(0)>-<A^{s}(t)><A^{s^{\prime}}(0)> \\
& C_{2}^{s s^{\prime}}(t)=\sum_{i}\left[<E_{i}^{s}(t) E_{i}^{s^{\prime}}(0)>-<E_{i}^{s}(t)><E_{i}^{s^{\prime}}(0)>\right],
\end{aligned}
$$

Although $<E_{i}^{s}(t)>$ vanishes for a lattice which is invarient under the cubic symmetry group acting on the space directions, one of the lattices we use is slightly asymmetric, $30 \times 32^{2}$. This lattice is close enough to symmetric, however, that the contribution of the disconnected term in $C_{2}^{s s^{\prime}}(t)$ turn out to be significantly smaller than our statistical errors.

For any pair of sizes $s$ and $s^{\prime}$, at sufficiently large values of $t$ and the lattice time direction periodicity $T$, the glueball propagators approach the asymptotic form

$$
C_{i}^{s s^{\prime}}(t) \rightarrow Z_{i}^{s s^{\prime}}\left\{\exp \left(-m_{i} t\right)+\exp \left[-m_{i}(T-t)\right]\right\}
$$

where $m_{0}$ is the energy eigenvalue of the lightest $0^{++}$zero-momentum state $\mid 0^{++}>, m_{2}$ is the common energy eigenvalue of the lightest $2^{++}$zero-momentum states $\mid 2^{++}, i>$ 
with spin index $i=1,2$, and $Z_{0}^{s s^{\prime}}$ and $Z_{2}^{s s^{\prime}}$ are related to the vacuum state $\mid \Omega>$ matrix elements

$$
\begin{aligned}
& Z_{0}^{s s^{\prime}}=<\Omega\left|A^{s}(t)\right| 0^{++}><0^{++}\left|A^{s^{\prime}}(t)\right| \Omega> \\
& Z_{2}^{s s^{\prime}}=\sum_{i}<\Omega\left|E_{i}^{s}(t)\right| 2^{++}, i><2^{++}, i\left|E_{i}^{s^{\prime}}(t)\right| \Omega>.
\end{aligned}
$$

It is not hard to show that, for any value of $t$, the propagators $C_{i}^{s s^{\prime}}(t)$ are sums of terms of the form in Eqs. (2.9) with each $Z_{0}^{s s^{\prime}}$ matrix hermitian and non-negative. Thus each propagator with the same size smearing at both ends, $C_{i}^{s s}(t)$, is a convex function. To extract a glueball mass from each propagator $C_{i}^{s s^{\prime}}(t)$, we search for a range of $s, s^{\prime}$ and $t$ for which the asymptotic form is approached. We then fit the asymptotic form to this data set, minimizing the fit's $\chi^{2}$ determined from the full correlation matrix of the target data set.

Statistical uncertainties are found for the resulting masses by the bootstrap method [9]. From each ensemble of $\mathrm{N}$ gauge field configurations, we generate a collection of 100 bootstrap ensembles. Each bootstrap ensemble consists of N configurations selected randomly from the underlying $\mathrm{N}$ member ensemble, allowing repeats. For each of the bootstrap ensembles, masses are determined by repeating our fitting method from the beginning but using the fitting interval already chosen for the full ensemble. The statistical uncertainty of a mass prediction is taken to be half the difference between a mass which is higher than all but $15.9 \%$ of the bootstrap masses and a mass which is lower than all but $15.9 \%$ of the bootstrap masses. In the limit of large $\mathrm{N}$, for which the collection of bootstrap masses will approach a gaussian distribution, the definition we use for statistical uncertainty approaches the dispersion, $d$, given by $d^{2}=<m^{2}>-<m>^{2}$.

Our method of determining masses and error bars is nearly the same as used in Ref. [4]. The sole difference is that in the calculation of Ref. [4] the fitted range of $s, s^{\prime}$ and $t$ is chosen independently on each different bootstrap data set to minimize the fit's value of $\chi^{2}$ per degree of freedom. Although allowing the fitted range of $s, s^{\prime}$ and $t$ to be chosen independently for each bootstrap ensemble appears to be logically the best procedure, we were forced to use a fixed fitting range in the present 
calculation. The statistical fluctuations in glueball propagators for some bootstrap data sets were sufficiently large that an automated choice of fitting range would be difficult to implement, while choosing fitting ranges by hand for each bootstrap ensemble would be too time consuming.

It is perhaps worth mentioning that the values of glueball masses obtained from Eqs. (2.9) are independent of the choice of the convergence parameter $r$. Coulomb gauge leaves the time direction link matrices unconstrainted. The integration over time direction link matrices in the path integral generates, in the corresponding time evolution transfer matrix of the Hilbert space formulation of lattice QCD [11, a projection operator onto the gauge invariant sector of Hilbert space. For values of $r$ which are large, the states created by our glueball operators will include large gauge variant components. These are canceled off by the time evolution projection operators to the gauge invariant sector of Hilbert space. The resulting propagators of Eqs. (2.8) exhibit only the masses of physical, gauge invariant states. The price of this cancellation is an increase in the statistical errors in masses. In practice we choose a small enough value of $r$ so that this increase in statistical errors is negligible.

In comparison to the gauge invarient smearing methods of Refs. [7, 8], the numerical cost of fixing to Coulomb gauge is rather large. As a results our method of constructing glueball propagators is less efficient that those of Refs. [7, 8]. An advantage of our procedure is that it does a more complete job of eliminating high frequency components of the gauge field than do the methods of Refs. [7, 8] and, correspondingly, is free of the potential problems discussed in Ref. [10. A comparison of our data with that of Ref. [5] also suggests that, for a fixed ensemble size, our method may give somewhat smaller statistical errors in glueball propagators at large time separations.

Our procedure for determining glueball masses from glueball propagators appears to be marginally more reliable than two competing methods. We found that fitting the full propagator matrix $C_{i}^{s s^{\prime}}(t)$ gives masses which are somewhat more stable to variations in ensemble size than either fitting the correlation function $C_{i}^{s s}(t)$ for a single optimal smearing size $s$, or fitting the correlation function $\sum_{s s^{\prime}} \zeta^{s} \zeta^{s^{\prime}} C_{i}^{s s^{\prime}}(t)$ for a single optimal vector $\zeta^{s}$ [2]. It also appears to us that a single fit to the full 
set of data provides the least biased way of resolving the small disagreements which occur among the masses found with various different combinations of smearing size for the propagator's source and sink.

\section{MASSES}

Gauge configurations were generated according to the standard Wilson one plaquette action using the Cabbibo-Marinari-Okawa algorithm. Table 1 lists the lattice sizes, $\beta=6 / g_{0}^{2}$ values, sweeps skipped between gauge configurations, and number of configurations used in the ensembles from which glueball propagators were calculated. Each configuration was equilibrated for at least 30000 sweeps before we began collecting data. The number of sweeps between configurations on which data was collected, however, was not large enough for us to be certain whether or not successive data samples were correlated. We therefore checked our bootstrap evaluation of error bars to be sure that it was not distorted by the presence of correlations. At $\beta$ of $5.7,6.17$ and 6.4 , our collections of gauge configurations were divided into sequential groups of 2, 4, 8 and 16 gauge configurations. Glueball propagators were averaged over each bin, and a bootstrap error evaluation was done on masses determined from the binned ensembles. Any correlations present in an ensemble of propagators before binning become progressively weaker in the ensembles with larger bins. Our error estimates showed no significant variation as bins were made larger. A direct calculation of the correlation between successive glueball propagators in the ensembles of Table [ also produced no statistically significant evidence for correlations.

For all the lattices listed in Table 1, the gauge fixing convergence parameter $r$ was set to $10^{-5}$. The average number of sweeps required for convergence to this accuracy ranged from 1525 on the lattice $24^{3} \times 36$ at $\beta$ of 5.93 , to 2270 on the lattice $16^{3} \times 32$ at $\beta$ of 5.7 .

To determine the range of smearing sizes and time separations from which to extract masses for each lattice and spin, we evaluated effective masses $m_{i}^{s}(t)$ by fitting propagators $C_{i}^{s s}(t)$ to Eqs. (2.9) at separations $t$ and $t+1$. In all cases, the time direction period $T$ was large enough and the time separation at which our mass 
signals disappeared into noise small enough that the values we found for $m_{i}^{s}(t)$ were equivalent to using the simpler definition $m_{i}^{s}(t) a=\ln \left[C_{i}^{s s}(t) / C_{i}^{s s}(t+1)\right]$, where $a$ is the lattice spacing. At large $t$ each $m_{i}^{s}(t)$ approaches the corresponding $m_{i}$ of Eqs. (2.9). It follows from the convexity of $C_{i}^{s s}(t)$ that this approach will be from above. A plateau at large $t$ in a graph of $m_{i}^{s}(t)$ identifies the region over which it is reasonable to try fitting propagator data to Eqs. (2.9). Figures (11) - (8) show effective masses for the $0^{++}$glueball on all lattices of Table 1 and for the $2^{++}$glueball on the three lattices at largest $\beta$. At time separations beyond those shown, we were unable to obtain statistically significant effective mass values. At the two lowest $\beta$ we were unable to obtain enough statistically significant effective mass values for the $2^{++}$glueball to find a plateau in the mass. In each case, we show the smearing size which gives the best signal. All of the data for the $0^{++}$glueball shows fairly clear effective mass plateaus extending over at least 4 time values. The data for the $2^{++}$ also shows effective mass plateaus, but not as clearly as the $0^{++}$data.

Having found effective mass plateaus for each smearing size, we fit the largest set of time slices which could be considered to be in the plateau to Eqs. (2.9) and determined the $\chi^{2}$ per degree of freedom of the best fit. We then removed a time slice at small $t$ and repeated the fit. In most cases, either the value of $\chi^{2}$ per degree of freedom of the best fit decreased or the fitted mass decreased by a statistically significant amount, showing that the narrower fitting range provided a better estimate of the lowest mass. We repeated the process as long as either the effective mass continued to decrease significantly or $\chi^{2}$ per degree of freedom decreased. The process was stopped when we found either a large increase in $\chi^{2}$ per degree of freedom or a large increase in the fitted mass's statistical uncertainty. We adopted the time window before either a large increase in $\chi^{2}$ or a large increase in statistical uncertainty as the best fitting range. In about half of the cases we considered, a fit to the window with one fewer time slice than the best choice showed a larger value of mass than the best choice. The convexity of the propagators with the same smearing size at both ends implies this increase can only occur as a result of statistical noise. The occurrence of an increase in fitted mass about half of the time suggests that, within statistical errors, we obtained the correct lowest mass in each case. Tables (2) and (3) show the 
masses and values of $\chi^{2}$ per degree of freedom obtained in the process of choosing fitting ranges for the data in Figures (1) - (8). For each sequence of fits in Tables (2) and (3), the fitting range next to last was selected as the best choice. The vertical lines in Figures (1) - (8) show the final fitting range and the horizontal lines show the mass found for this range. In some cases the fitting range extends beyond the last time slice for which an effective mass is given since, by convention, we assign each effective mass to the smaller of the two time values from which it has been determined. Tables (4), (5), and (6) show the masses found by fitting, on the best fitting ranges, propagators with the same size smearing at both ends. For each $\beta$ and spin, the optimal fitting range determined according to our criteria turned out to the be same for each smearing size for which we had a reliable signal. This was not put in as a constraint, is slightly surprising, and is probably a statistical accident.

Final mass values for each particle were determined by making a combined fit, as described earlier, to data for the full set of different smearing sizes for which we had reliable masses. The results of the final fits are shown in Tables (7) and (8). For the $2^{++}$glueball, at $\beta$ of 6.17 , the combined data set dictated by our selection rule consisted of smearing sizes 4,5 and 6 over the range from time separation 2 to time separation 5. This fit gave an unacceptable $\chi^{2}$ per degree of freedom of 10.6. Removing the data at time separation 2 and fitting only from separations 3 to 5 reduces $\chi^{2}$ of 1.2 and gives the mass shown in Table (8).

In addition to our calculations of propagators and masses for the $0^{++}$and $2^{++}$ glueballs, we also calculated propagators for the $1^{+-}$glueball. The effective mass plateaus in these propagators were weak, however, and the masses which could be extracted were close to the expected masses for torelon pairs. Thus the reliability of the predicted $1^{+-}$masses was not clear, and we do not report these results here.

\section{INFINITE VOLUME, CONTINUUM LIMITS}

The quantity $z=m_{0++} L$, where $L$ is the lattice period, for the masses in Table (7) ranges from 12.45 , using the smaller period of 30 on the lattice $30 \times 32^{2} \times 40$ at $\beta$ of 6.4 , to 19.46 , on the lattice $24^{3} \times 36$ at $\beta$ of 5.93 . Masses calculated in a large 
finite volume approach their values in infinite volume according to [12]

$$
m_{i}(z)=m_{i}(\infty)\left[1-g_{i} \frac{\exp \left(\frac{-\sqrt{3}}{2} z\right)}{z}\right] .
$$

For $m_{0}$ the coefficient $g_{0}$ has been estimated [13] to be $190 \pm 70$. Thus our values for $m_{0}$ should differ from their infinite volume values by less than $0.05 \%$. For $m_{2}$ it appears from data in Ref. [13 that $g_{2}<2000$. The range of $z$ we use then gives $m_{2}$ which agree with infinite volume values to better than $0.4 \%$. Thus all of the masses shown in Tables (7) and (8) differ from their values in infinite volume by amounts which are negligible in comparision to the statistical uncertainties.

In preparation for evaluating the continuum limit of $m_{0}$ and $m_{2}$ in physical units, the values we calculated for $m_{i} a$ in lattice units were rescaled by $\Lambda_{\frac{(0)}{M S}} a$ in lattice units. According to mean-field improved lattice perturbation theory [14, 15]

$$
\frac{1}{g_{\overline{M S}}^{2}}=\frac{<\operatorname{Tr} U / 3>}{g^{2}}+0.025
$$

where $g^{2}=6 / \beta$ is the lattice coupling constant, and $\langle\operatorname{Tr} U\rangle$ is the average value of a plaquette. Then with $\alpha \overline{M S}=g_{\overline{M S}}^{2} / 4 \pi$, the solution to the two loop Callan-Symanzik equation for $\alpha \overline{M S}(\pi / a)$ with zero flavors of quark vacuum polarization gives

$$
\begin{aligned}
\Lambda_{\overline{M S}}^{(0)} a & =\pi\left(\frac{b_{1} \alpha \overline{M S}}{1+b_{1} \alpha_{\overline{M S}}}\right)^{-\frac{b_{1}}{b_{0}}} \exp \left(-\frac{1}{b_{0} \alpha_{\overline{M S}}}\right) \\
b_{0} & =\frac{11}{2 \pi} \\
b_{1} & =\frac{51}{22 \pi} .
\end{aligned}
$$

Values of $\alpha \frac{\overline{M S}}{\text { and }} \Lambda_{\frac{(0)}{M S}}$ a determined from Eqs. (4.2) and (4.3) are given in Table (9). Values of $m_{i} / \Lambda_{\overline{M S}}^{(0)}$ determined from Tables (7), (8) and (9) are shown in Figures (9) and (10). In Ref. [4] it was found that the rho mass ratio $m_{\rho} / \Lambda_{\frac{(0)}{M S}}$ is nearly independent of $\beta$ and within $2 \%$ of its continuum limit once $\beta \geq 5.7$. Thus graphs of our glueball masses scaled by the rho mass, $m_{i} / m_{\rho}$, would differ from Figures (9) and (10) only by a constant rescaling of the vertical axis. 
Since the leading irrelevant term in the Wilson action for pure gauge fields is $O\left(a^{2}\right)$, we expect that for sufficiently small lattice spacing mass ratios will approach their continuum limits according to

$$
\frac{m_{i}}{\Lambda_{\frac{(0)}{M S}}}(a)=\frac{m_{i}}{\Lambda_{\overline{M S}}^{(0)}}(0)+c_{i} a^{2},
$$

with $a$-independent coefficients $c_{i}$. Fits of $m_{0}$ to Eq. (4.4) using five data points, using the four data points with the smallest $a$ and using the three data points with the smallest $a$ are listed in Table (10). Since Eq. (4.4) is an asymptotic form approached at small lattice spacing $a$, and since the $\chi^{2}$ per degree of freedom of fits to Eq. (4.4) progressively decreases as data points with larger $a$ are eliminated, it appears that the fit using three data points gives the most reliable extrapolation to the continuum limit. It is interesting to notice that a quadratic fit to all five data points gives essentially the same continuum limit and same $\chi^{2}$ per degree of freedom as the linear fit at the three smallest points. For $m_{2}$ we have data at only the three smallest values of $a$. A fit of Eq. (4.4) to these, giving a fairly small $\chi^{2}$ is also shown in Table (10). The three point fits for $m_{0}$ and $m_{2}$ are shown in Figures (9) and (10), respectively. The vertical lines at $a=0$ in each figure are the statistical uncertainties in the extrapolated mass values.

To obtain masses in physical units from the continuum limit ratios $m_{i} / \Lambda \frac{(0)}{M S}$, we use the continum value of $245.0 \pm 9.2 \mathrm{MeV}$ for $\Lambda \frac{(0)}{M S}$ found in Ref. 四 from the continuum limit of $m_{\rho} / \Lambda \frac{(0)}{M S}$ combined with the observed value of $m_{\rho}$. For $m_{0}$ we get $1340 \pm 160 \mathrm{MeV}$, and for $m_{2}$ we find $1900 \pm 320 \mathrm{MeV}$, with statistical uncertainties combining the uncertainties in $m_{i} / \Lambda_{\overline{M S}}^{(0)}$ and in $\Lambda \frac{(0)}{M S}$.

An alternate way to find the continuum limit of lattice values of $m_{i} a$ is to divide each by the square root of the string tension in lattice units, $\sqrt{K} a$, and extrapolate $m_{i} / \sqrt{K}$ to its continuum limit. The continuum limit of $\sqrt{K}$, however, is not a directly observable quantity. Its continuum value can be determined by extrapolating $\sqrt{K} / m_{\rho}$ to its continuum limit, then using the observed value of $m_{\rho}$. The resulting continuum prediction for $\sqrt{K}$ inherits statistical errors both from lattice calculations of $\sqrt{K}$ and from lattice calculations of $m_{\rho}$. The continuum value which we have used for $\Lambda \frac{(0)}{M S}$, 
on the other hand, has statistical errors only from lattice calculations of $m_{\rho}$. Thus the method we have adopted should give a smaller uncertainty for continuum mass predictions in physical units. The central value of the answers obtained extrapolating $m_{i} / \sqrt{K}$ do not appear to be significantly different from our present answers. For example, interpolating published values of $\sqrt{K} a$ [16] to our values of $\beta$, using these to find the continuum limit of $m_{0} / \sqrt{K}$, and assuming the popular value of $440 \mathrm{MeV}$ for continuum $\sqrt{K}$, gives a continuum $m_{0}$ of about $1310 \mathrm{MeV}$.

As mentioned in Sect. (四), continuum limit predictions for $m_{0}$ of $1550 \pm 50 \mathrm{MeV}$ and for $m_{2}$ of $2270 \pm 100 \mathrm{MeV}$ were reported recently in Ref. [5]. These numbers were obtained by taking $m_{0} / \sqrt{K}$ and $m_{2} / \sqrt{K}$ at $\beta$ of 6.4 as continuum values. If the fit in Ref. [5] of $m_{0} / \sqrt{K}$ to a linear function of $K a^{2}$ is actually extrapolated to the continuum, the central value of $m_{0}$ becomes $1600 \mathrm{MeV}$. This number differs from our result by about 1.6 standard deviations. It appears to us, however, that the central values of both predictions quoted in Ref. [5] may be somewhat higher and the error bars on both predictions somewhat smaller than the data of Ref. [5] itself suggests.

For $m_{0}$ and $m_{2}$ at $\beta$ of 6.4, Ref. [5] takes the effective masses found from the $0^{++}$and $2^{++}$propagators between time separations 2 and 3 . From the effective mass tables given in Ref. [5] it seems unclear how close the effective masses at these time separations are to their asymptotic large time separation values and how reliable the error estimates on these effective masses may be. The central values of effective masses found from the $0^{++}$and $2^{++}$propagators fall monotonically at time separations larger than 2. Both effective masses between time separations of 4 and 5, for example, are about 10\% lower than corresponding numbers between separations 2 and 3 and have error bars which are larger than the corresponding errors by factors of more than 2 . It also seems to us uncertain whether the $m_{0}$ value which Ref. [5] takes from Ref. [2] for $\beta$ of 6.2 is a reliable estimate of the asymptotic effective mass at large time separation. Our calculation of $m_{0}$ at $\beta$ of 6.17 , which should lie above $m_{0}$ at 6.2 , is actually lower by about $10 \%$. The data of Ref. [2] for $m_{0}$ at $\beta$ of 5.9 is consistent with our fit of $m_{0}$ to a linear function of $\left[\Lambda \frac{(0)}{M S} a\right]^{2}$, while $m_{2}$ is again above the extrapolation of our data.

The results of Refs. [5] and [2] become closer to ours if $m_{0}$ and $m_{2}$ are taken 
from effective masses at larger time separations approximating the ranges over which our mass fits were done. Combining effective masses between time separations 2 and 3 at $\beta$ of 5.9 and 6.0 [2], time separations 3 and 4 at $\beta$ of 6.2 [2], and time separations 4 and 5 at $\beta$ of 6.4 [5], we fit $m_{i} / \Lambda \frac{(0)}{M S}$ to linear functions of $\left[\Lambda \frac{(0)}{M S} a\right]^{2}$. The continuum $m_{0} / \Lambda \frac{(0)}{M S}$ becomes $6.11 \pm 0.52$ and $m_{2} / \Lambda \frac{(0)}{M S}$ becomes $7.75 \pm 0.89$. With $\Lambda \frac{(0)}{M S}$ of $245.0 \pm 9.2 \mathrm{MeV}$, the continuum prediction for $m_{0}$ is $1500 \pm 140 \mathrm{MeV}$ and for $m_{2}$ is $1900 \pm 230 \mathrm{MeV}$. These results are both less than one standard deviation from our corresponding predictions. A fit to this set of data combined with ours in the region $\beta \geq 5.9$ gives a continuum limit for $m_{0} / \Lambda \frac{(0)}{M S}$ of $5.89 \pm 0.38$ and for $m_{2} / \Lambda \frac{(0)}{M S}$ of $7.77 \pm 0.73$. These values yield $m_{0}$ of $1440 \pm 110 \mathrm{MeV}$ and $m_{2}$ of $1900 \pm 190 \mathrm{MeV}$.

We would like to thank Frank Butler for writing some of the analysis software which we used, and Mike Cassera, Molly Elliott, Dave George, Chi Chai Huang and Ed Nowicki for their work on GF11.

\section{References}

[1] B. Berg and A. Billoire, Nucl. Phys. B221 (1983) 109.

[2] C. Michael and M. Teper, Nucl. Phys. B314 (1989) 347.

[3] D. H. Weingarten, Phys. Lett. 109B (1982) 57; Nucl. Phys. B215 [FS7] (1983) 1.

[4] F. Butler, H. Chen, J. Sexton, A. Vaccarino and D. Weingarten, Phys. Rev. Letts. 70 (1993) 2849; Nucl. Phys. B (Proc. Suppl. ) 30 (1993) 377.

[5] G. Bali, et al. Wuppertal Preprint WUB 93-10, to appear in Phys. Lett. B.

[6] D. Weingarten, Nucl. Phys. B (Proc. Suppl. ) 17 (1990) 272.

[7] L. Griffiths, C. Michael, and P. Rakow, Phys. Lett. B129 (1983) 351.

[8] M. Teper, Phys. Lett. 183B (1986) 345. 
[9] B. Efron, The Jacknife, the Bootstrap and Other Resampling Plans (Society for Industrial and Applied Mathematics, Philadelphia, 1982).

[10] A. Kronfeld and P. Van Ball, Nucl. Phys. (Proc. Suppl. ) 9 (1989) 227.

[11] M. Lüscher, Comm. Math. Phys. 54 (1977) 283.

[12] M. Lüscher, Comm. Math. Phys. 104 (1986) 177.

[13] G. Schierholz, Nucl. Phys. (Proc. Suppl. ) 9 (1989) 244.

[14] G. P. Lepage and P. B. Mackenzie, Fermilab preprint Fermilab-Pub-91/353-T (1992).

[15] A. X. El-Khadra, G. H. Hockney, A. S. Kronfeld and P. B. Mackenzie, Phys. Rev. Letts. 69 (1992) 729.

[16] S. Peratonis and C. Michael, Nucl. Phys. B347 (1990) 854; K. D. Born et al. Nucl. Phys. B (Proc. Suppl. ) 20 (1991) 394; G. S. Bali and K. Schilling, Nucl. Phys. B (Proc. Suppl. ) 30 (1993) 513.

[17] P. de Forcrand, et al. Phys. Letts. 152B (1985) 107. 


\begin{tabular}{|r|c|c|c|}
\hline lattice & $\beta$ & skip & count \\
\hline $16^{3} \times 24$ & 5.70 & 400 & 4039 \\
$20^{3} \times 30$ & 5.83 & 400 & 4002 \\
$24^{3} \times 36$ & 5.93 & 400 & 4004 \\
$30 \times 32^{2} \times 40$ & 6.17 & 400 & 2005 \\
$30 \times 32^{2} \times 40$ & 6.40 & 400 & 2002 \\
\hline
\end{tabular}

Table 1: Configurations analyzed.

\begin{tabular}{|c|c|c|c|c|}
\hline$\beta$ & size & range & mass & $\chi^{2}$ \\
\hline 5.70 & 2 & $1-5$ & $0.958 \pm 0.023$ & 0.56 \\
5.70 & 2 & $2-5$ & $0.896 \pm 0.058$ & 0.20 \\
5.70 & 2 & $3-5$ & $0.978 \pm 0.176$ & 0.03 \\
\hline 5.83 & 2 & $1-5$ & $0.896 \pm 0.017$ & 0.93 \\
5.83 & 2 & $2-5$ & $0.845 \pm 0.039$ & 0.62 \\
5.83 & 2 & $3-5$ & $0.798 \pm 0.076$ & 0.92 \\
\hline 5.93 & 2 & $1-5$ & $0.856 \pm 0.015$ & 1.15 \\
5.93 & 2 & $2-5$ & $0.833 \pm 0.033$ & 1.54 \\
5.93 & 2 & $3-5$ & $0.871 \pm 0.085$ & 2.78 \\
\hline 6.17 & 4 & $2-7$ & $0.564 \pm 0.028$ & 1.55 \\
6.17 & 4 & $3-7$ & $0.482 \pm 0.035$ & 0.20 \\
6.17 & 4 & $4-7$ & $0.461 \pm 0.058$ & 0.20 \\
\hline 6.40 & 5 & $3-9$ & $0.444 \pm 0.029$ & 1.12 \\
6.40 & 5 & $4-9$ & $0.387 \pm 0.035$ & 0.34 \\
6.40 & 5 & $5-9$ & $0.399 \pm 0.050$ & 0.43 \\
\hline
\end{tabular}

Table 2: Masses, in units of $1 / a$, obtained for $0^{++}$glueballs over various fitting ranges. 


\begin{tabular}{|c|c|c|c|c|}
\hline$\beta$ & size & range & mass & $\chi^{2}$ \\
\hline 5.93 & 4 & $1-4$ & $1.223 \pm 0.050$ & 0.03 \\
5.93 & 4 & $2-4$ & $1.197 \pm 0.188$ & 0.03 \\
5.93 & 4 & $3-4$ & $1.288 \pm 0.766$ & \\
\hline 6.17 & 5 & $1-5$ & $0.871 \pm 0.029$ & 0.22 \\
6.17 & 5 & $2-5$ & $0.852 \pm 0.061$ & 0.28 \\
6.17 & 5 & $3-5$ & $0.835 \pm 0.137$ & 0.55 \\
\hline 6.40 & 5 & $2-8$ & $0.579 \pm 0.023$ & 1.25 \\
6.40 & 5 & $3-8$ & $0.632 \pm 0.053$ & 1.09 \\
6.40 & 5 & $4-8$ & $0.522 \pm 0.059$ & 0.49 \\
6.40 & 5 & $5-8$ & $0.641 \pm 0.128$ & 0.04 \\
\hline
\end{tabular}

Table 3: Masses, in units of $1 / a$, obtained for $2^{++}$glueballs over various fitting ranges.

\begin{tabular}{|c|c|c|c|c|}
\hline$\beta$ & size & range & mass & $\chi^{2}$ \\
\hline 5.70 & 0 & $2-5$ & $0.985 \pm 0.141$ & 0.01 \\
5.70 & 1 & $2-5$ & $0.948 \pm 0.064$ & 0.54 \\
5.70 & 2 & $2-5$ & $0.896 \pm 0.058$ & 0.20 \\
5.70 & 3 & $2-5$ & $0.842 \pm 0.072$ & 0.57 \\
5.70 & 4 & $2-5$ & $0.797 \pm 0.103$ & 0.87 \\
\hline 5.83 & 1 & $2-5$ & $0.889 \pm 0.056$ & 2.02 \\
5.83 & 2 & $2-5$ & $0.845 \pm 0.039$ & 0.62 \\
5.83 & 3 & $2-5$ & $0.840 \pm 0.047$ & 1.30 \\
5.83 & 4 & $2-5$ & $0.845 \pm 0.075$ & 1.36 \\
5.83 & 5 & $2-5$ & $0.834 \pm 0.116$ & 0.79 \\
5.83 & 6 & $2-5$ & $0.798 \pm 0.105$ & 1.00 \\
\hline
\end{tabular}

Table 4: $0^{++}$glueball masses, in units of $1 / a$, fitted to optimal ranges for each size of smearing. 


\begin{tabular}{|c|c|c|c|c|}
\hline$\beta$ & size & range & mass & $\chi^{2}$ \\
\hline 5.93 & 1 & $2-5$ & $0.905 \pm 0.053$ & 0.13 \\
5.93 & 2 & $2-5$ & $0.833 \pm 0.031$ & 1.54 \\
5.93 & 3 & $2-5$ & $0.796 \pm 0.030$ & 3.19 \\
5.93 & 4 & $2-5$ & $0.781 \pm 0.043$ & 3.98 \\
5.93 & 5 & $2-5$ & $0.746 \pm 0.060$ & 2.41 \\
5.93 & 6 & $2-5$ & $0.724 \pm 0.090$ & 1.52 \\
\hline 6.17 & 2 & $3-7$ & $0.504 \pm 0.055$ & 0.83 \\
6.17 & 3 & $3-7$ & $0.505 \pm 0.035$ & 0.37 \\
6.17 & 4 & $3-7$ & $0.482 \pm 0.035$ & 0.20 \\
6.17 & 5 & $3-7$ & $0.473 \pm 0.041$ & 0.04 \\
6.17 & 6 & $3-7$ & $0.495 \pm 0.060$ & 0.30 \\
\hline 6.40 & 3 & $4-9$ & $0.392 \pm 0.043$ & 0.38 \\
6.40 & 4 & $4-9$ & $0.389 \pm 0.037$ & 0.27 \\
6.40 & 5 & $4-9$ & $0.387 \pm 0.035$ & 0.34 \\
6.40 & 6 & $4-9$ & $0.392 \pm 0.034$ & 0.52 \\
6.40 & 7 & $4-9$ & $0.400 \pm 0.036$ & 0.66 \\
6.40 & 8 & $4-9$ & $0.408 \pm 0.042$ & 0.77 \\
\hline
\end{tabular}

Table 5: $0^{++}$glueball masses, in units of $1 / a$, fitted to optimal ranges for each size of smearing. 


\begin{tabular}{|c|c|c|c|c|}
\hline$\beta$ & size & range & mass & $\chi^{2}$ \\
\hline 5.93 & 3 & $2-4$ & $1.131 \pm 0.109$ & 0.11 \\
5.93 & 4 & $2-4$ & $1.197 \pm 0.188$ & 0.03 \\
5.93 & 5 & $2-4$ & $1.464 \pm 0.405$ & 0.19 \\
\hline 6.17 & 4 & $2-5$ & $0.842 \pm 0.059$ & 0.18 \\
6.17 & 5 & $2-5$ & $0.852 \pm 0.062$ & 0.28 \\
6.17 & 6 & $2-5$ & $0.887 \pm 0.080$ & 0.22 \\
\hline 6.40 & 4 & $4-8$ & $0.514 \pm 0.066$ & 0.41 \\
6.40 & 5 & $4-8$ & $0.522 \pm 0.059$ & 0.49 \\
6.40 & 6 & $4-8$ & $0.510 \pm 0.062$ & 0.81 \\
\hline
\end{tabular}

Table 6: $2^{++}$glueball masses, in units of $1 / a$, fitted to optimal ranges for each size of smearing.

\begin{tabular}{|c|c|c|c|c|}
\hline$\beta$ & sizes & range & mass & $\chi^{2}$ \\
\hline 5.70 & $0-4$ & $2-5$ & $0.928 \pm 0.058$ & 1.35 \\
\hline 5.83 & $1-6$ & $2-5$ & $0.858 \pm 0.043$ & 1.80 \\
\hline 5.93 & $1-6$ & $2-5$ & $0.811 \pm 0.033$ & 2.70 \\
\hline 6.17 & $2-6$ & $3-7$ & $0.489 \pm 0.031$ & 1.29 \\
\hline 6.40 & $3-8$ & $4-9$ & $0.415 \pm 0.043$ & 1.68 \\
\hline
\end{tabular}

Table 7: $0^{++}$glueball masses, in units of $1 / a$, obtained from fits to propagator matrices for sets of several smearing sizes. 


\begin{tabular}{|c|c|c|c|c|}
\hline$\beta$ & sizes & range & mass & $\chi^{2}$ \\
\hline 5.93 & $3-5$ & $2-4$ & $1.144 \pm 0.107$ & 2.58 \\
\hline 6.17 & $4-6$ & $3-5$ & $0.816 \pm 0.119$ & 1.17 \\
\hline 6.40 & $4-6$ & $4-8$ & $0.504 \pm 0.061$ & 2.22 \\
\hline
\end{tabular}

Table 8: $2^{++}$glueball masses, in units of $1 / a$, obtained from fits to propagator matrices for sets of several smearing sizes.

\begin{tabular}{|r|c|c|c|}
\hline lattice & $\beta$ & $\alpha_{\overline{M S}}$ & $\Lambda_{\overline{M S}} a$ \\
\hline $16^{3} \times 24$ & 5.70 & 0.1456 & 0.1661 \\
$20^{3} \times 30$ & 5.83 & 0.1370 & 0.1329 \\
$24^{3} \times 36$ & 5.93 & 0.1318 & 0.1144 \\
$30 \times 32^{2} \times 40$ & 6.17 & 0.1218 & 0.08265 \\
$30 \times 32^{2} \times 40$ & 6.40 & 0.1141 & 0.06177 \\
\hline
\end{tabular}

Table 9: Values of $\alpha \overline{M S}$ and $\Lambda_{\overline{M S}} a$. Statistical uncertainties are all smaller than 1 in the last decimal place.

\begin{tabular}{|c|c|c|c|c|}
\hline$J^{P C}$ & fit & points & $m_{i} / \Lambda_{\overline{M S}}$ & $\chi^{2}$ \\
\hline $0^{++}$ & linear & 3 & $5.46 \pm 0.64$ & 2.36 \\
$0^{++}$ & linear & 4 & $6.19 \pm 0.50$ & 2.89 \\
$0^{++}$ & linear & 5 & $6.97 \pm 0.29$ & 3.43 \\
$0^{++}$ & quadratic & 5 & $5.37 \pm 0.81$ & 2.26 \\
$2^{++}$ & linear & 3 & $7.76 \pm 1.26$ & 0.47 \\
\hline
\end{tabular}

Table 10: Extrapolations of $m_{i} / \Lambda_{\overline{M S}}$ to the continuum limit using either linear or quadratic functions of $\left[\Lambda_{\overline{M S}} a\right]^{2}$. 


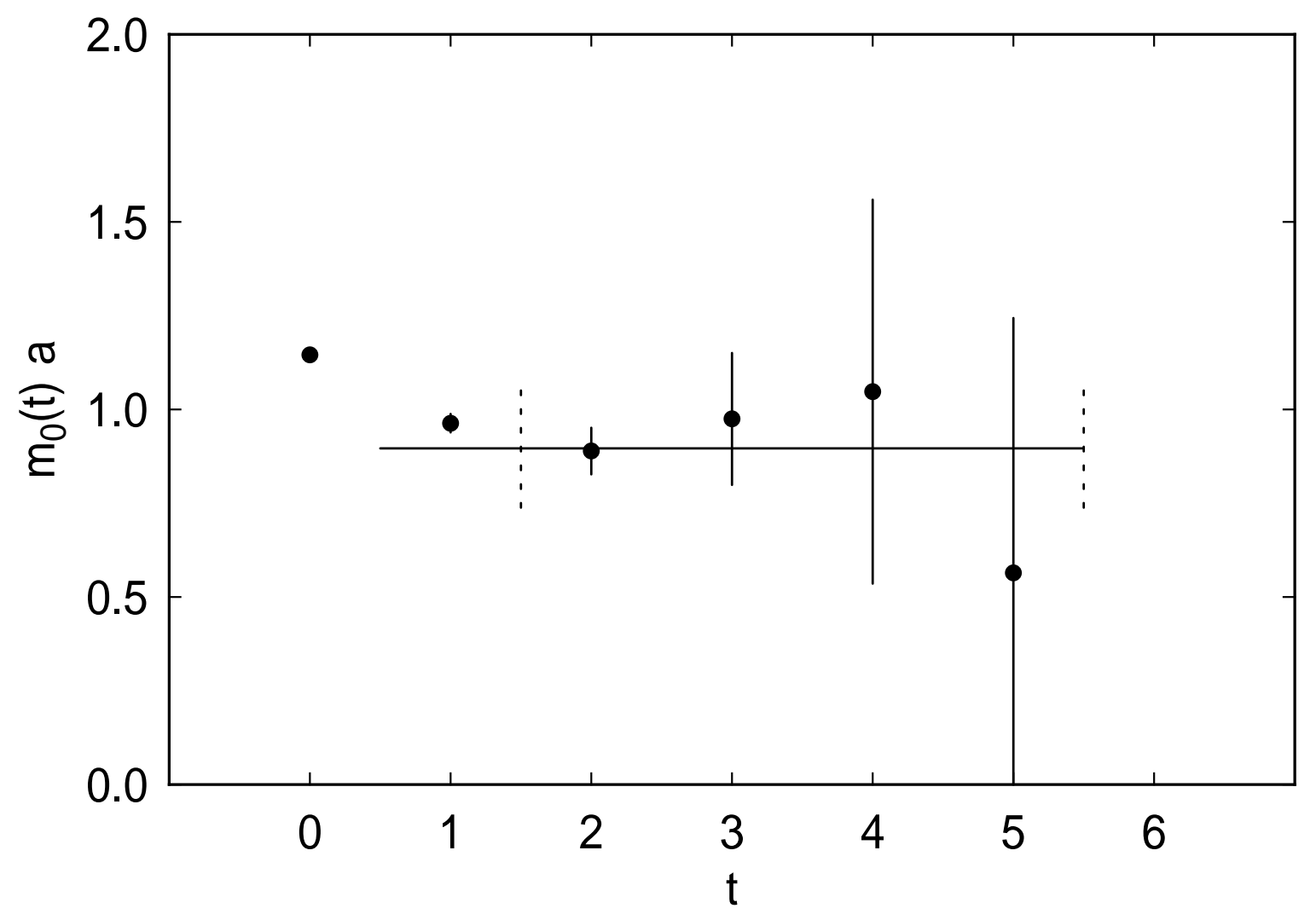

Figure 1: Effective masses and fitted mass for the $0^{++}$glueball on a $16^{3} \times 24$ lattice at $\beta=5.7$ for smearing size 2 . 


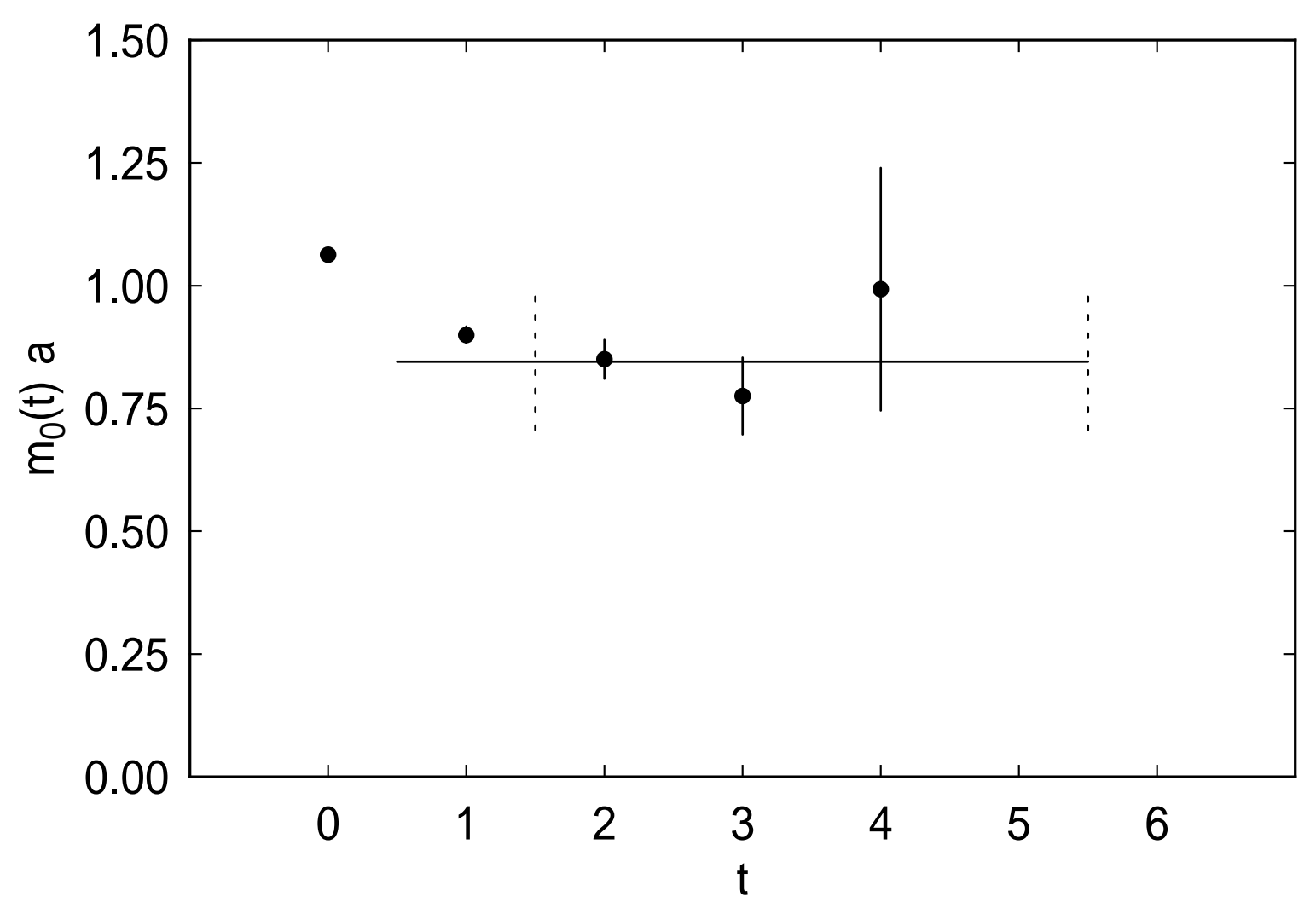

Figure 2: Effective masses and fitted mass for the $0^{++}$glueball on a $20^{3} \times 30$ lattice at $\beta=5.83$ for smearing size 2 . 


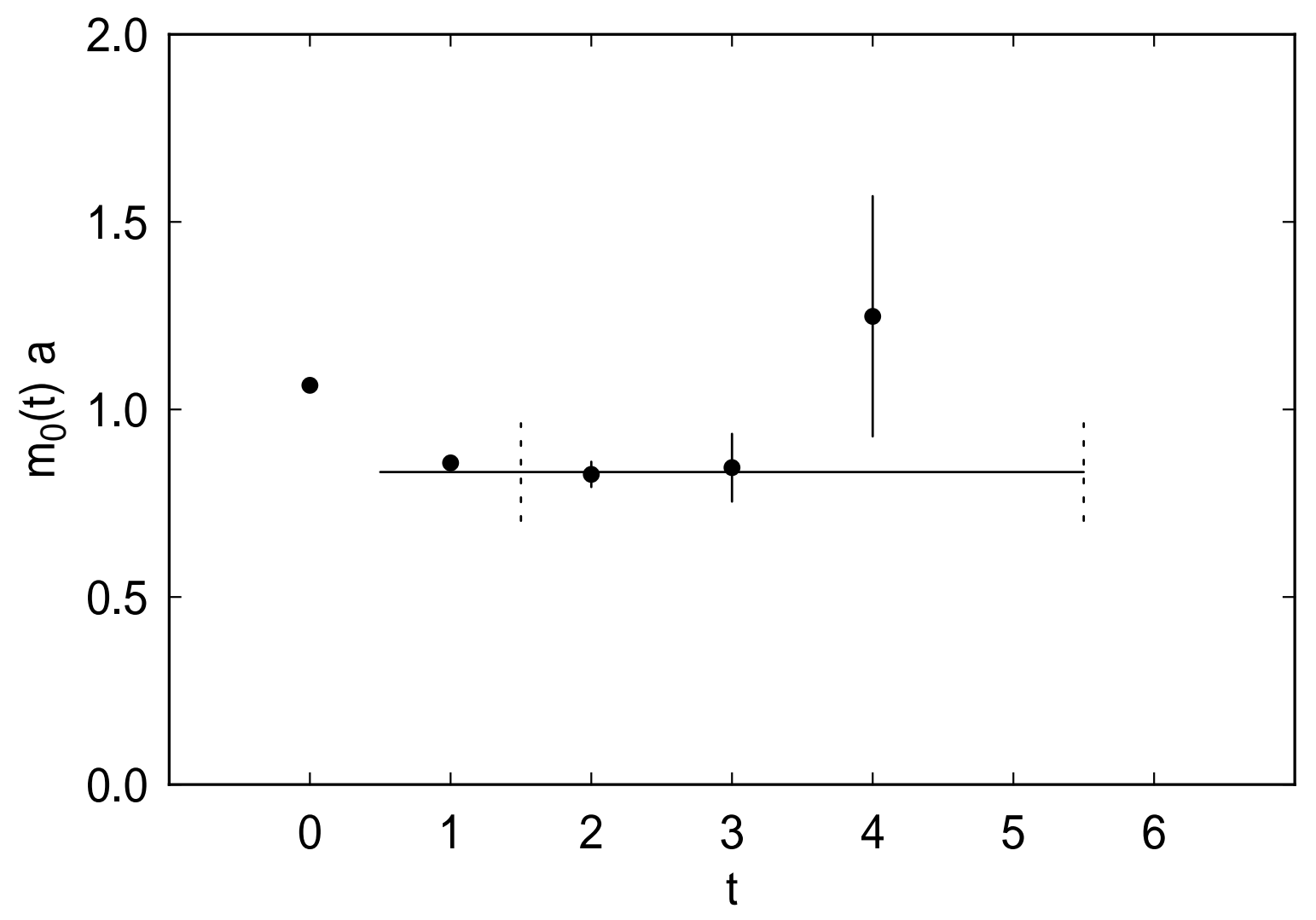

Figure 3: Effective masses and fitted mass for the $0^{++}$glueball on a $24^{3} \times 36$ lattice at $\beta=5.93$ for smearing size 2 . 


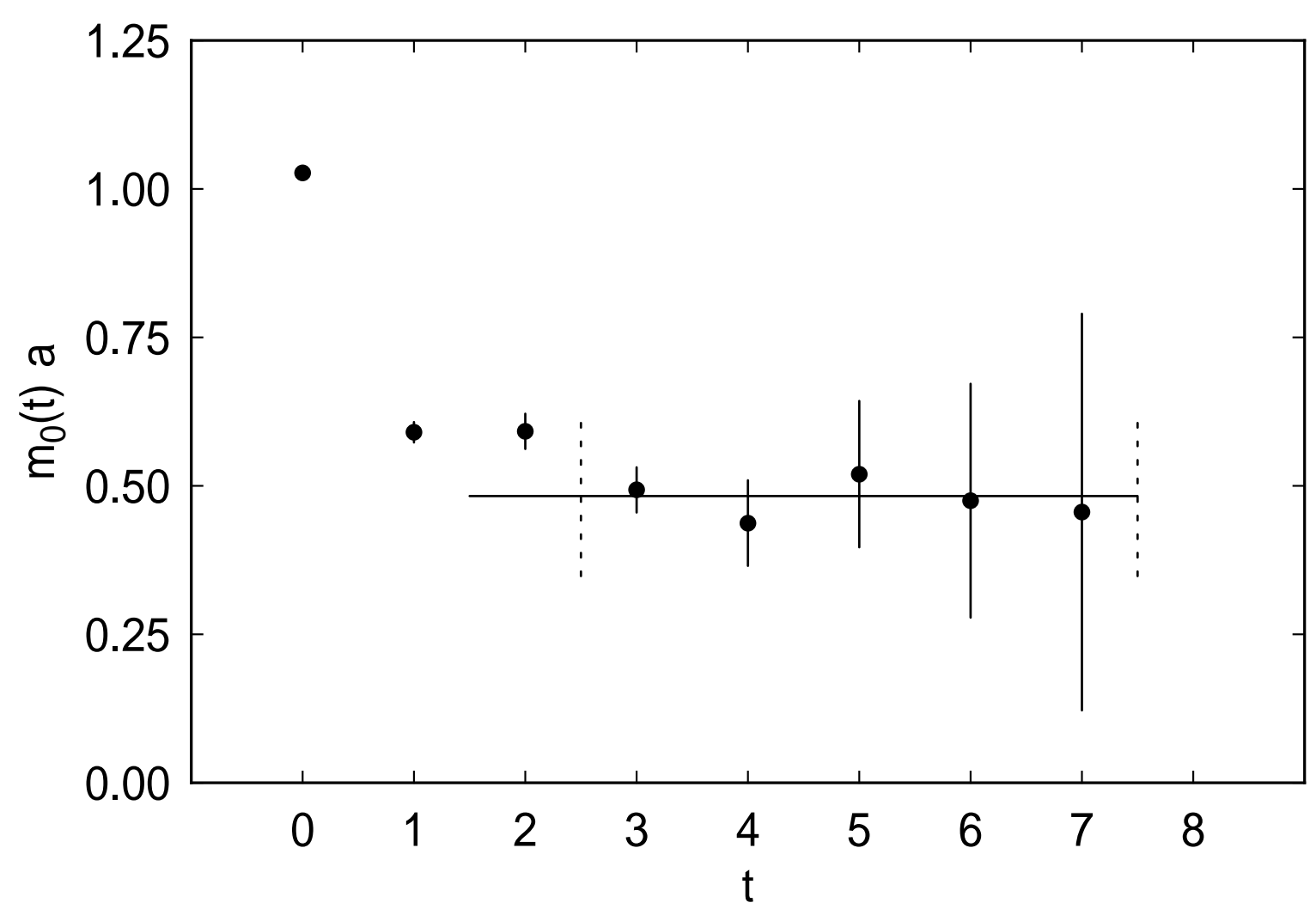

Figure 4: Effective masses and fitted mass for the $0^{++}$glueball with smearing size 4 on a $30 \times 32^{2} \times 40$ lattice at $\beta=6.17$. 


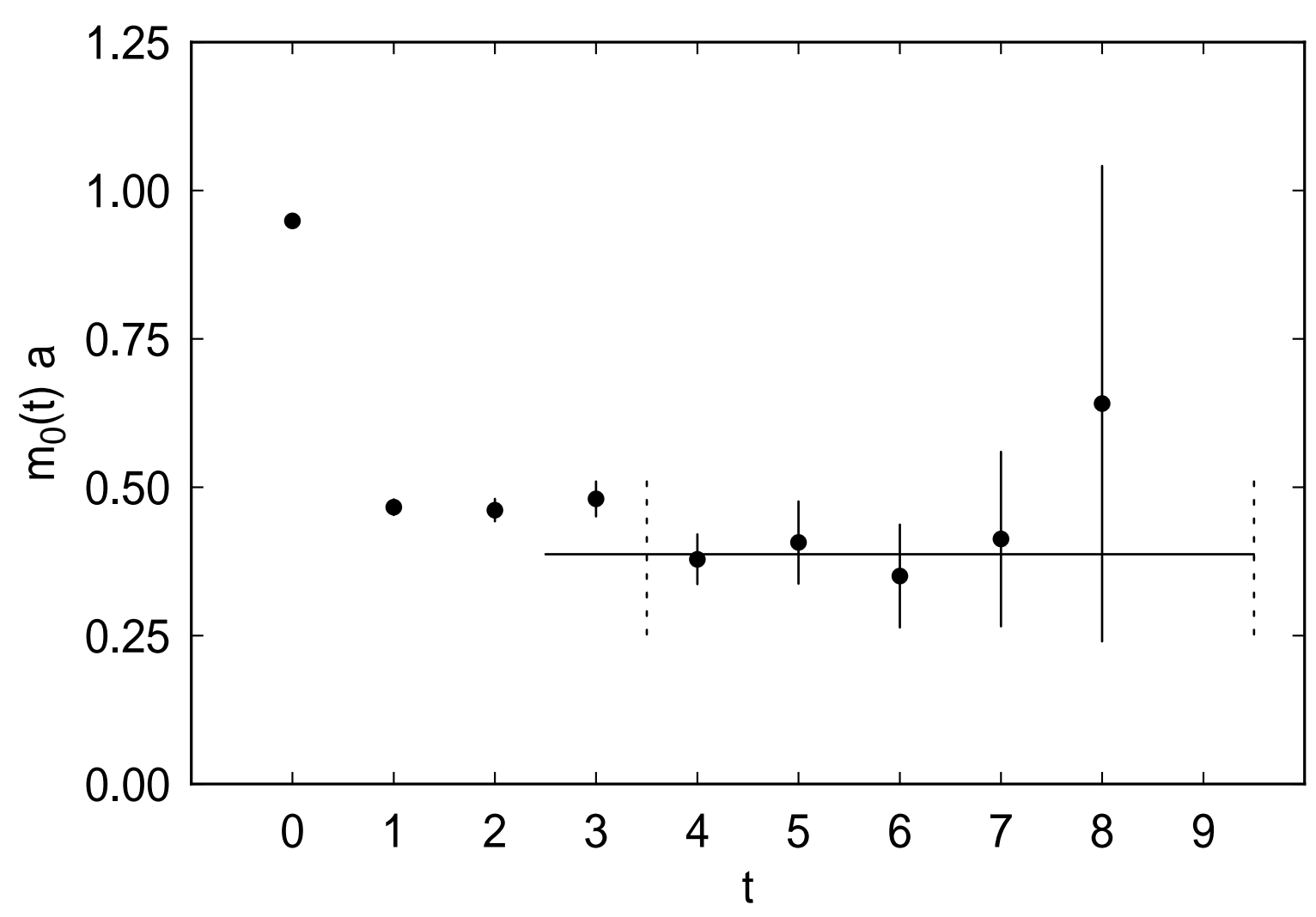

Figure 5: Effective masses and fitted mass for the $0^{++}$glueball with smearing size 5 on a $30 \times 32^{2} \times 40$ lattice at $\beta=6.40$. 


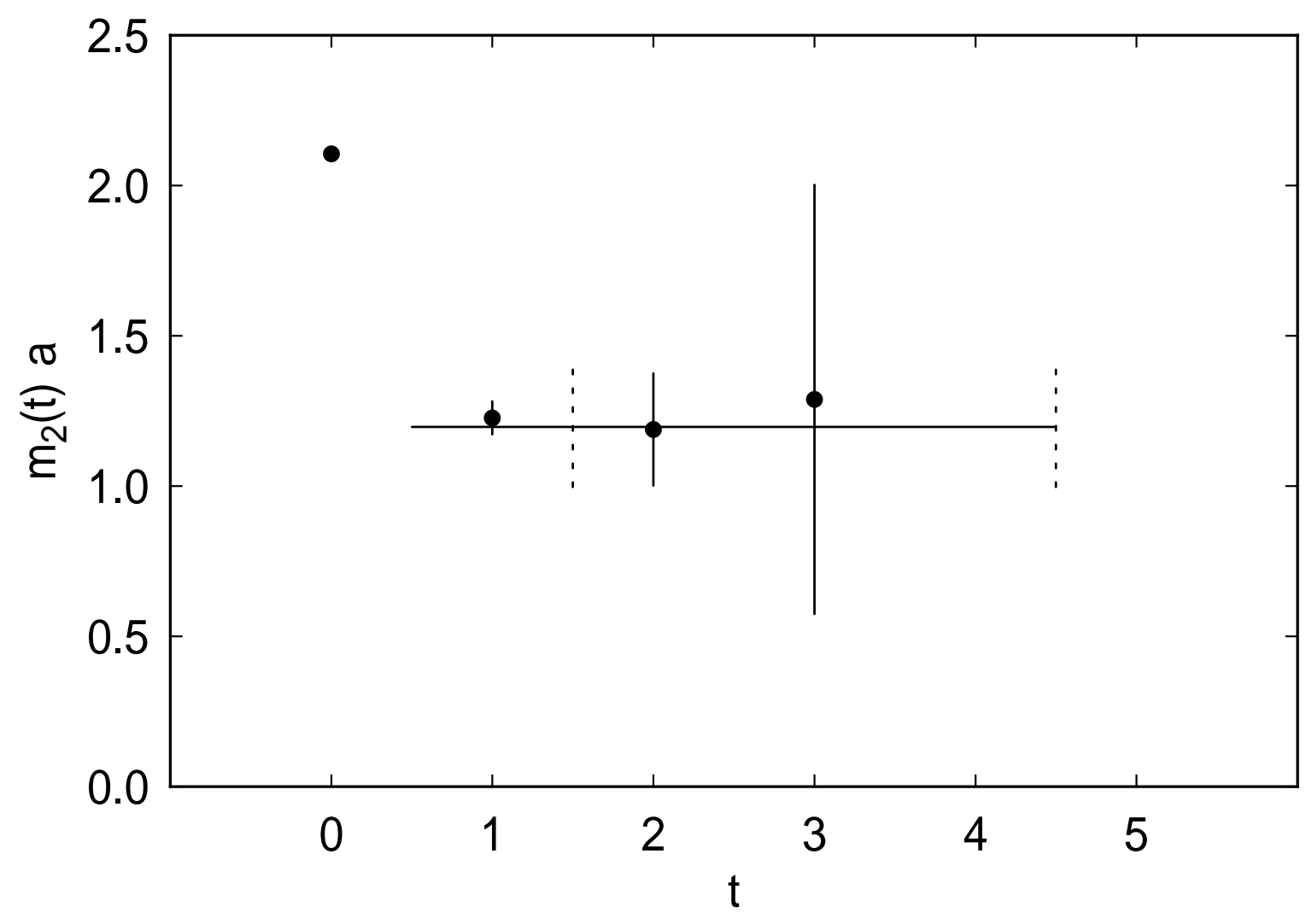

Figure 6: Effective masses and fitted mass for the $2^{++}$glueball with smearing size 4 on a $24^{3} \times 36$ lattice at $\beta=5.93$. 


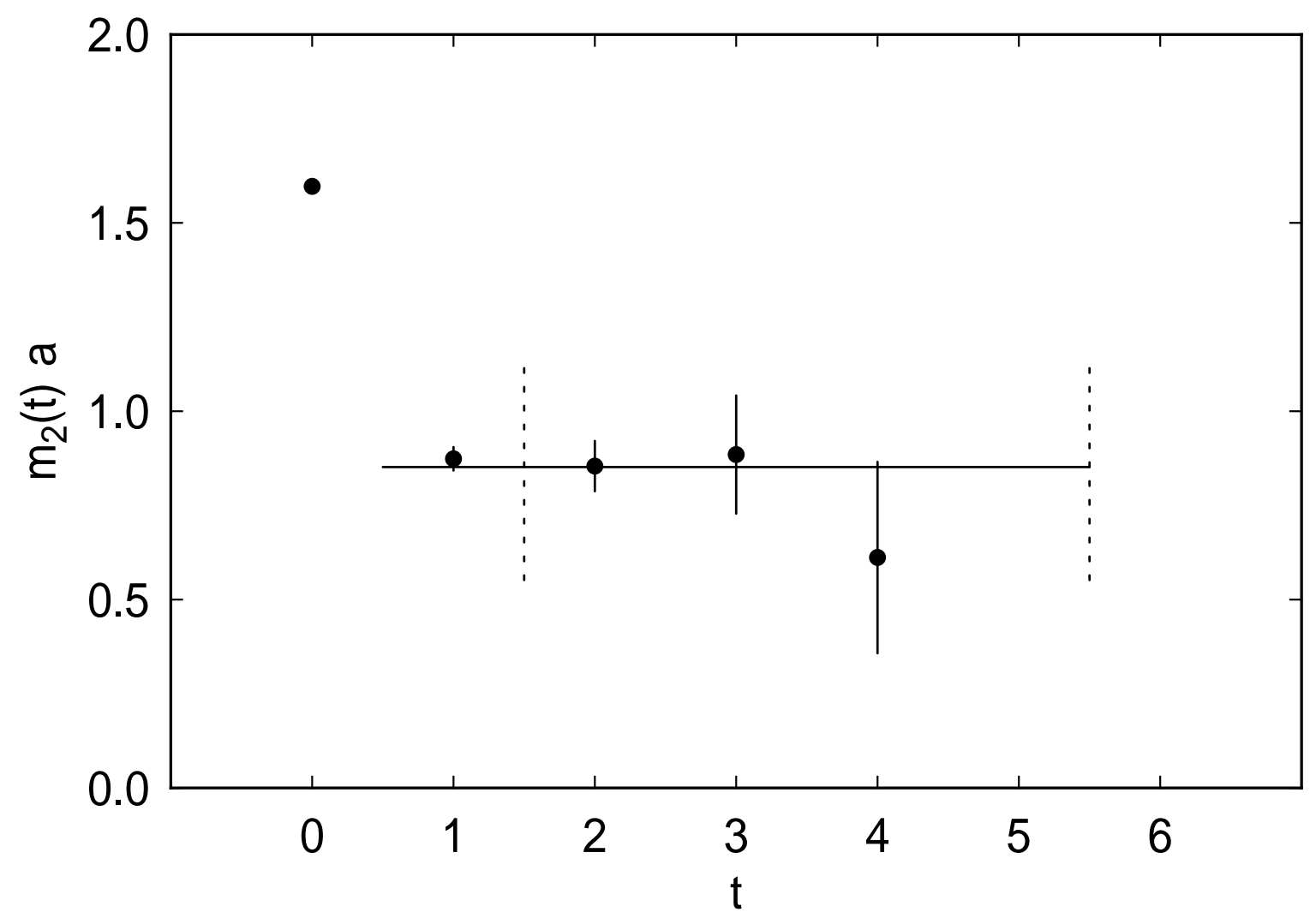

Figure 7: Effective masses and fitted mass for the $2^{++}$glueball with smearing size 5 on a $30 \times 32^{2} \times 40$ lattice at $\beta=6.17$. 


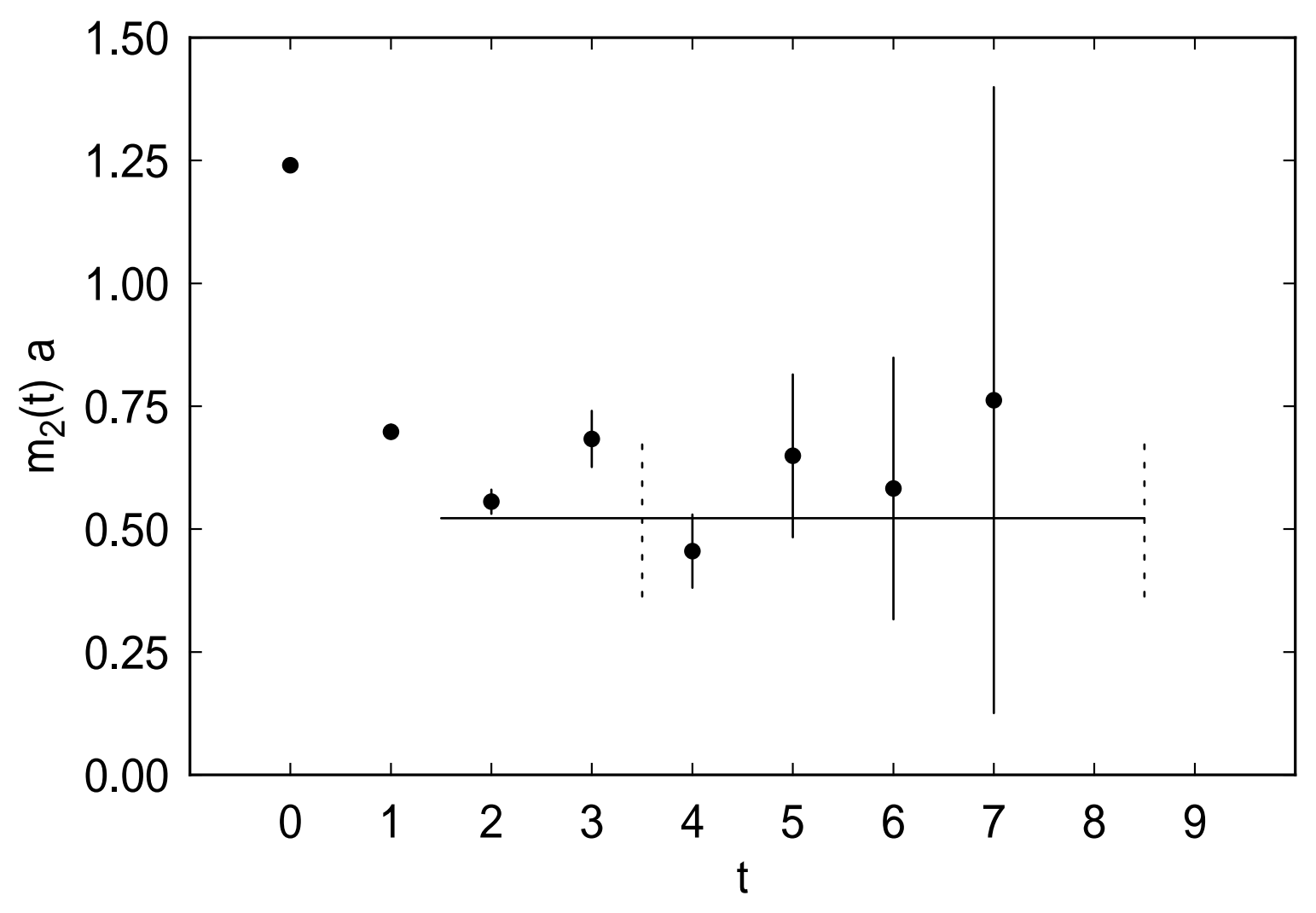

Figure 8: Effective masses and fitted mass for the $2^{++}$glueball with smearing size 5 on a $30 \times 32^{2} \times 40$ lattice at $\beta=6.40$. 


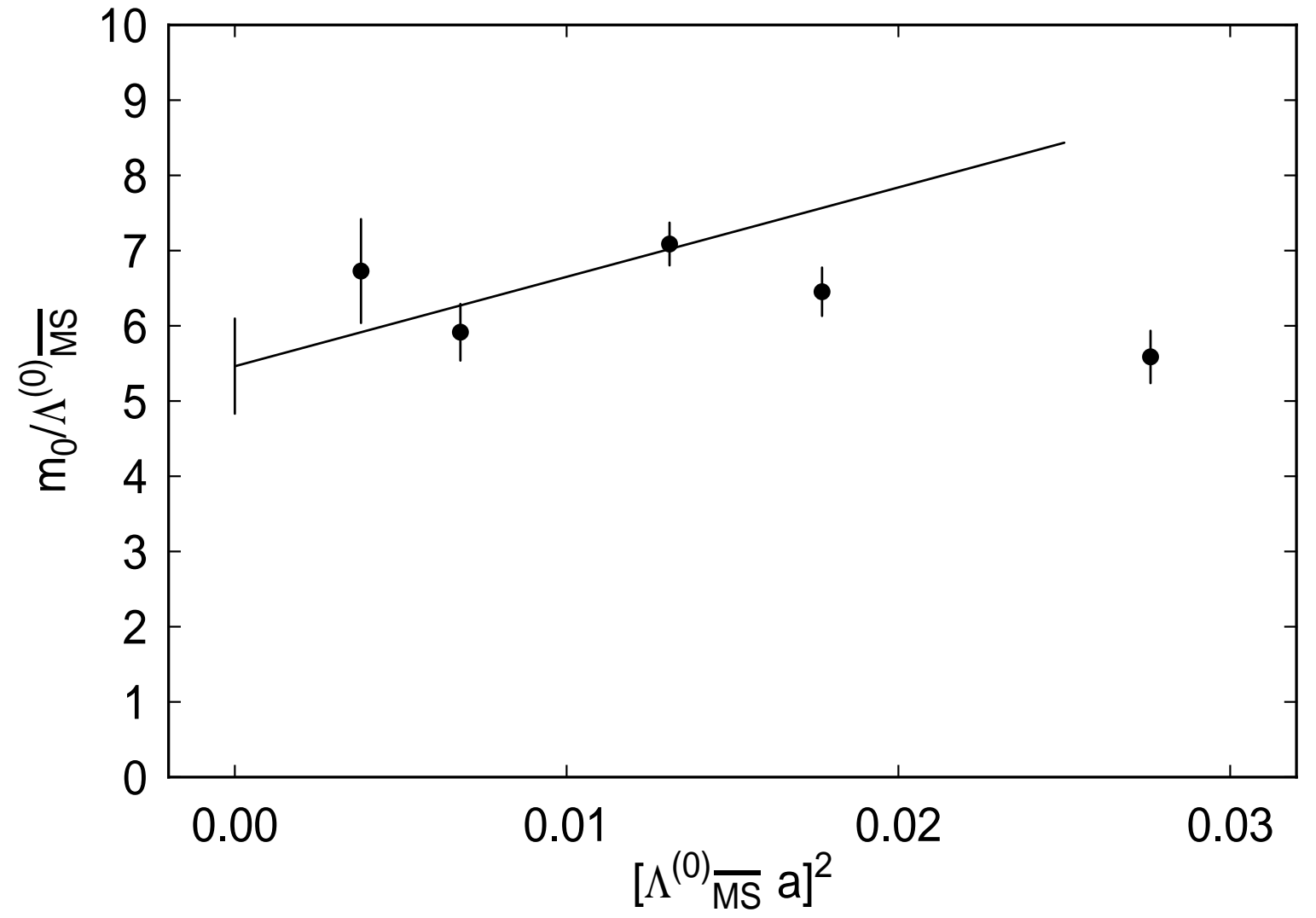

Figure 9: Values of $m_{0} / \Lambda_{\frac{(0)}{M S}}$ as a function of $\left[\Lambda \frac{(0)}{M S} a\right]^{2}$ and linear extrapolation to $a=0$. 


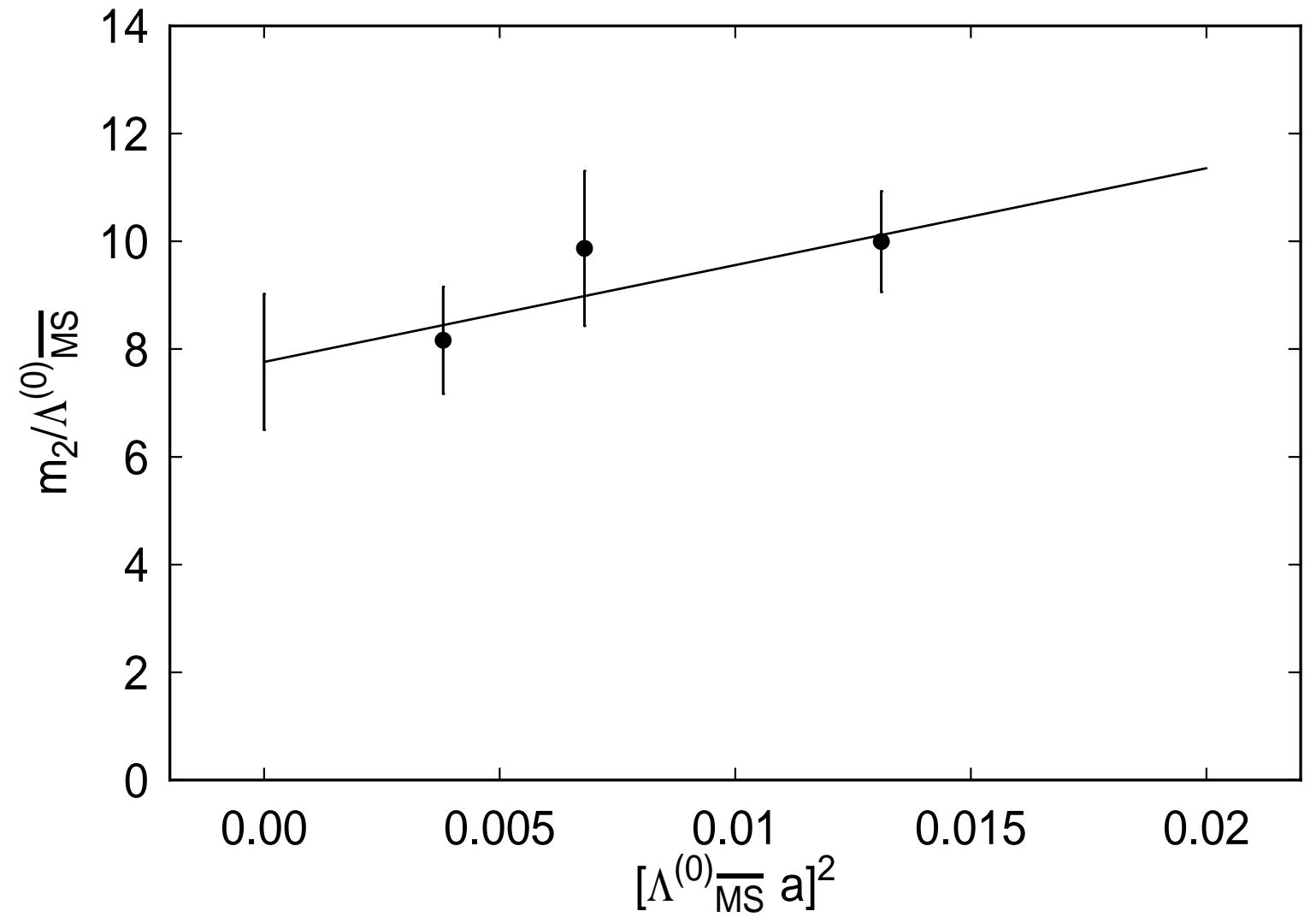

Figure 10: Values of $m_{2} / \Lambda_{\frac{(0)}{M S}}$ as a function of $\left[\Lambda \frac{(0)}{M S} a\right]^{2}$ and linear extrapolation to $a=0$. 